\title{
Synthesis and study of new antimicrobial benzothiazoles substituted on heterocyclic ring
}

\author{
Ivica Sigmundová, Pavol Zahradník, ${ }^{*}$ Peter Magdolen, and Helena Bujdáková \\ Faculty of Natural Sciences, Comenius University, 84104 Bratislava, Slovakia \\ E-mail:zahradnik@fns.uniba.sk
}

Dedicated to Professor Arlette Soladié-Cavallo on the occasion of her 70th birthday

\begin{abstract}
New 2-styryl benzothiazolium salts substituted on the heterocyclic ring have been synthesised by the condensation of 3-alkyl-2-methylbenzothiazolium halides with 4-substituted benzaldehydes. The intramolecular charge transfer from the electron-donor substituent to the benzothiazolium ring is a typical feature of the prepared compounds. This type of benzothiazolium derivatives can be used as pharmaceutical substances as well as compounds with nonlinear optical response. Antimicrobial in vitro activity was determined and the influence of substituents has been evaluated. The substituents on the heterocyclic ring in positions 5 and 6 do not increase the biological activity significantly.
\end{abstract}

Keywords: Benzothiazolium salts, antimicrobial activity, synthesis

\section{Introduction}

Some of the benzothiazole derivatives with a push-pull structure (conjugated system with donor and acceptor end groups) are well known pharmaceutical substances ${ }^{1}$ as well as compounds suitable as nonlinear optical materials, molecular dyads and chemosensors. ${ }^{2,3}$ The bactericidal properties of 2-substituted benzothiazoles have been recognized for a long time, the most effective structures were recognized as 3-allyl and 3-propargyl benzothiazolium salts with $p$ dialkylaminostyryl substituents. ${ }^{4}$

This work is a part of the systematic study of biologically active benzothiazole derivatives. ${ }^{4,5}$ Previously, these compounds have been tested against model organism Euglena gracilis as well as other microorganisms and QSAR study has been carried out. ${ }^{6}$ A new series of benzothiazoles have been designed and synthesised. The structural features connected with the higher activity have been recognized as, 
1. quaternary nitrogen atom in the benzothiazole;

2. electron donor group $\mathrm{Y}$ in the $p$-position of the phenyl ring;

3. allyl, propargyl or methyl group at the heterocyclic nitrogen.

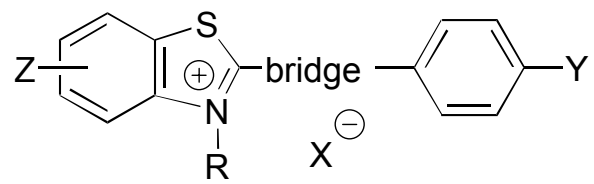

In previous studies we had modified the substituent $\mathrm{Y}$, alkyl $\mathrm{R}$ at the heterocyclic nitrogen as well as the type and the length of the bridge. The compounds with considerably higher activity had been designed and prepared. ${ }^{7}$ Less attention has been paid to the influence of substituent $\mathrm{Z}$ in the benzene part of the heterocycle. The original set of 91 compounds contained 4 compounds with $\mathrm{Cl}$ in position 4 , seven compounds with $6-\mathrm{Cl}$, four compounds with $4-\mathrm{CH}_{3}$ and eight with 6- $\mathrm{CH}_{3} .{ }^{4}$ The calculated Free-Wilson activity contributions of these substituents are not very high $\left(6-\mathrm{Cl}=0.206 ; 6-\mathrm{CH}_{3}=0.235 ; 4-\mathrm{CH}_{3}=0.250 ; 4-\mathrm{Cl}=0.260\right)$. The biological activity of compounds substituted on heterocyclic ring is increased in all cases.

In order to get a better understanding of the influence of this type of substituents, we decided to enlarge the studied systems to the derivatives bearing other substituents in position 5 and 6 of the heterocyclic ring. The aim of this paper is:

a) synthesis of 5- and 6-substituted benzothiazolium salts of the structure

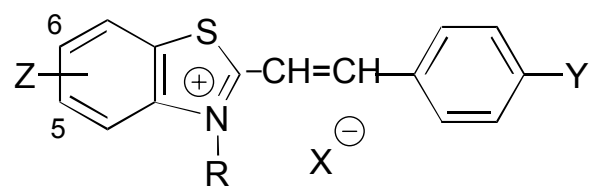

b) testing the 5-and 6-substituted benzothiazolium salts against selected Gram-positive bacteria as well as yeast microorganisms

c) evaluation of the Z-substituent effect on the structural parameters (UV-vis spectra, push-pull character and charge transfer) and biological properties of the compounds under study

\section{Results and Discussion}

In a previous paper we studied the electronic structure of some benzothiazole derivatives by the semiempirical quantum chemical methods. ${ }^{8}$ The calculations for 2-methylbenzothiazole showed the global negative charge in a 5-membered ring and positive charge in a 6-membered ring. The value of charge density in position 6 is more negative then in C-5 carbon and that is the reason of electrophilic attack at C-6. Thus the direct nitration of 2-methylbenzothiazole gives 2-methyl-6nitrobenzothiazole (1). ${ }^{9} \quad$ As the preparation of 5-nitroderivatives by direct nitration of 
benzothiazole ring is not possible, we prepared the 2-methyl-5-nitrobenzothiazole (2) indirectly via heterocycle 5-membered ring closure starting from 3-nitroaniline. ${ }^{10,11}$ The reduction of $\mathbf{1}$ and 2 with the $\mathrm{Fe} / \mathrm{HCl}$ under sonochemical conditions ${ }^{12}$ followed by the reaction with acetic anhydride provides corresponding acetylaminoderivatives 3 and $\mathbf{4}$ (Scheme 1).

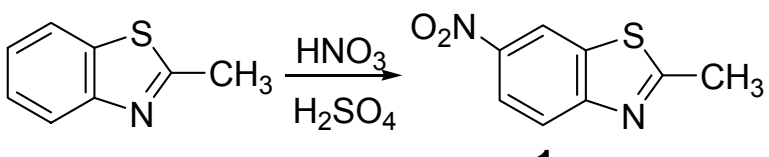

1
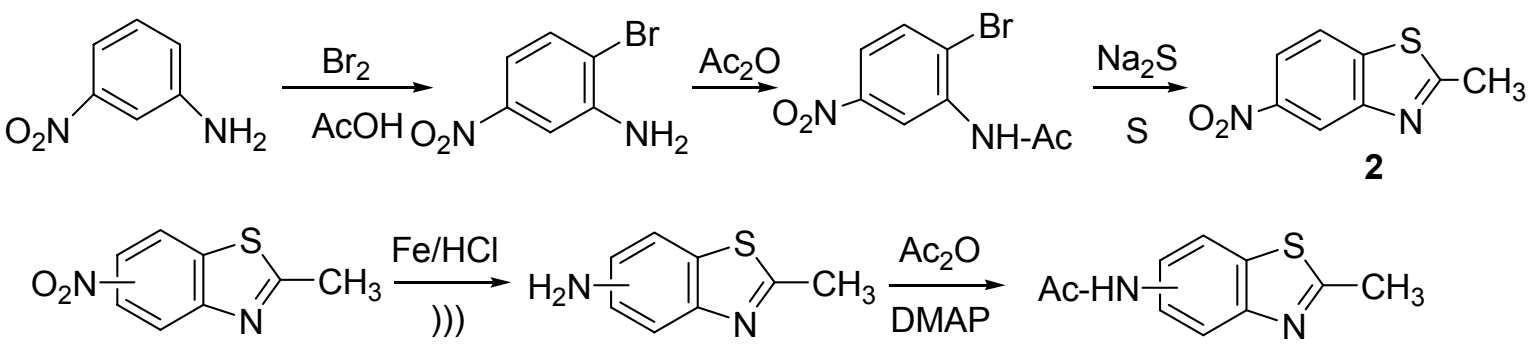

3,4

\section{Scheme 1}

Quaternization of the compounds 3 and $\mathbf{4}$ with alkyl bromides gave the appropriate benzothiazolium bromides $\mathbf{5 b}, \mathbf{5 c}, \mathbf{6 b}, \mathbf{6 c}$ in good yields (60-64\%) while quaternization of less reactive nitroderivatives $\mathbf{1}$ and $\mathbf{2}$ required microwave conditions to give nitrobenzothiazolium iodides 5a and 6a. ${ }^{13}$ The final 2-styrylbenzothiazolium salts (7a-7e, 8a- 8f) were prepared by the condensation reaction of appropriate 3-alkyl-2-methylbenzothiazol-3-ium bromides $(\mathbf{5 b}, \mathbf{5 c}, \mathbf{6 b}$, 6c) or iodides $(\mathbf{5 a}, \mathbf{6 a})$ with $p$-substituted benzaldehydes. ${ }^{14}$ The quaternary nitrogen atom in 2methylbenzothiazolium salts activates the hydrogens of the 2- $\mathrm{CH}_{3}$ group and therefore condensation reactions with benzaldehydes proceeded much faster and more efficiently than the condensations of the corresponding neutral molecules ${ }^{15,16}$ (Scheme 2). 


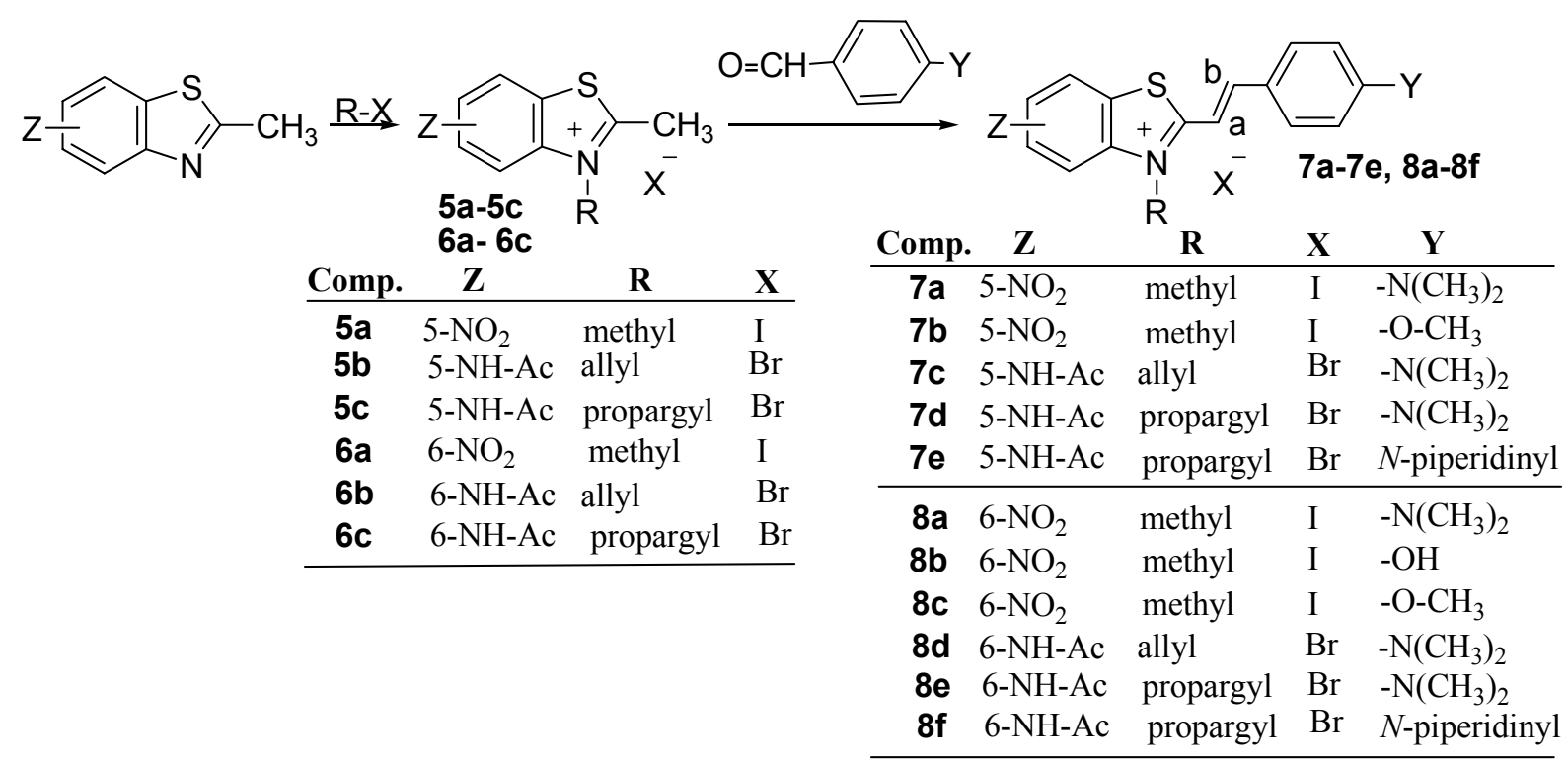

\section{Scheme 2}

All of the prepared final compounds have push-pull structure characterized by the electrondonor and electron-acceptor groups interacting through $\pi$-conjugated spacer. The important charge-transfer from the electron-donor substituent to the benzothiazolium ring is manifested by the long-wave band in visible region of UV-vis spectrum. According to these spectra the effects of amino and $\mathrm{OH}$ groups on the charge-transfer band are larger than the similar effect of methoxy group and they result in bathochromic shift of this absorption band. The withdrawing effect of benzothiazolium ring is enhanced by the $\mathrm{NO}_{2}$ substituent in the position 6 (compound $\mathbf{8 a}$ and $\mathbf{8 b}$ ). On the other hand the $\lambda_{\max }$ of the compounds with $\mathrm{NO}_{2}$ in position 5- and $\mathrm{CH}_{3}-\mathrm{CO}-$ $\mathrm{NH}$ - group in the position 5-, or 6- of the heterocycle are shifted to lower values.

\section{Experimental Section}

General Procedures. Melting points were determined with a Kofler apparatus and are uncorrected. ${ }^{1} \mathrm{H}$ NMR spectra were recorded on a Varian Gemini spectrometer (VNMRJ 1.1 D) at $300 \mathrm{MHz}$ in DMSO- $d_{6}$ with tetramethylsilane as internal standard. Chemical shifts are given in ppm ( $\delta$-scale), coupling constant $(J)$ in $\mathrm{Hz}$. The ${ }^{13} \mathrm{C}$ NMR spectra were recorded at $75 \mathrm{MHz}$ in DMSO- $d_{6}$. The UV-vis spectra were measured using a Hewlett-Packard Diode array spectrophotometer $8452 \mathrm{~A}$ in methanol. Wavelengths $\left(\lambda_{\max }\right)$ are given in nm, molar absorptivities $\left(\varepsilon_{\max }\right)$ in $1 \mathrm{~mol}^{-1} \mathrm{~cm}^{-1}$. Elemental analyses were determined on Carlo-Erba 1016 instrument. Microwave reactions were conducted using a microwave synthetizer Initiator ${ }^{\mathrm{TM}}$ Biotage with power range 0-300 $\mathrm{W}$ and pressure range 0-2.0 $\mathrm{MPa}$ in glass microwave vials sealed with septum. 
2,3-Dimethyl-5-(or 6-)nitrobenzothiazol-3-ium iodide (5a, 6a) was prepared under microwave conditions according to procedure described in literature. ${ }^{13}$

General procedure for synthesis of 5-(or 6-)acetylamino-2-methyl-3-alkylbenzothiazolium bromides $(5 b, 5 c, 6 b, 6 c)$

Excess of allyl or propargyl bromide $(1 \mathrm{ml})$ was added to the solution of 5- or 6-acetylamino-2methylbenzothiazole $(3,4)(0.5 \mathrm{~g}, 2.4 \mathrm{mmol})$ in $1 \mathrm{ml}$ nitromethane. The reaction mixture was heated to $80-85^{\circ} \mathrm{C}$ for $8-10 \mathrm{~h}$, then cooled and the resulting precipitate was filtered and washed with dry acetone.

5-Acetylamino-3-allyl-2-methylbenzothiazol-3-ium bromide (5b). Yield 60\%. M.p. 194-195 ${ }^{\circ} \mathrm{C} .{ }^{1} \mathrm{H}$ NMR (300 MHz, DMSO) $\delta 10.72$ (s, 1H, NH), 8.65 (s, 1H, H-4), 8.37 (d, J=9.0 Hz, 1H, $\mathrm{H}-7), 7.82(\mathrm{~d}, J=9.0 \mathrm{~Hz}, 1 \mathrm{H}, \mathrm{H}-6), 6.07$ (ddt, $\left.J=16.4,10.1,5.1 \mathrm{~Hz}, 1 \mathrm{H}, \mathrm{C} \underline{\mathrm{H}}=\mathrm{CH}_{2}\right), 5.40\left(\mathrm{~d}, J_{\text {cis }}\right.$ $=10.4 \mathrm{~Hz}, 1 \mathrm{H},=\mathrm{C} \underline{H H}), 5.35-5.30\left(\mathrm{~m}, 3 \mathrm{H}, \mathrm{N}-\mathrm{CH}_{2},=\mathrm{CH} \underline{\mathrm{H}}\right), 3.20\left(\mathrm{~s}, 3 \mathrm{H}, \mathrm{CH}_{3}\right), 2.16(\mathrm{~s}, 3 \mathrm{H}$, $\left.\mathrm{COCH}_{3}\right) .{ }^{13} \mathrm{C}$ NMR $(75 \mathrm{MHz}, \mathrm{DMSO}) \delta 177.67,169.24,141.17,140.42,128.94,124.76,122.66$, 120.10, 119.75, 105.23, 51.12, 24.04, 16.85. Analysis: Calcd (\%) for $\mathrm{C}_{13} \mathrm{H}_{15} \mathrm{BrN}_{2} \mathrm{OS}$ : C, 47.71; H, 4.62 N, 8.56; S, 9.83. Found: C, 47.60; H, 4.56; N, 8.49; S, 9.83.

5-Acetylamino-2-methyl-3-propargylbenzothiazol-3-ium bromide (5c). Yield 64\%. M.p. 187.5-189 ${ }^{\circ} \mathrm{C} .{ }^{1} \mathrm{H}$ NMR (300 MHz, DMSO) $\delta 10.70$ (s, 1H, NH), 8.78 (d, J=1.2 Hz, 1H, H-4), 8.37 (d, $J=9.0 \mathrm{~Hz}, 1 \mathrm{H}, \mathrm{H}-7), 7.81$ (dd, $J=9.0,1.8 \mathrm{~Hz}, 1 \mathrm{H}, \mathrm{H}-6), 5.63$ (d, $J=2.1 \mathrm{~Hz}, 2 \mathrm{H}, \mathrm{CH}_{2}$ ), $3.85(\mathrm{t}, J=2.1 \mathrm{~Hz}, 1 \mathrm{H}, \equiv \mathrm{CH}), 3.24\left(\mathrm{~s}, 3 \mathrm{H}, \mathrm{CH}_{3}\right), 2.16\left(\mathrm{~s}, 3 \mathrm{H}, \mathrm{COCH}_{3}\right) .{ }^{13} \mathrm{C} \mathrm{NMR}(75 \mathrm{MHz}$, DMSO) $\delta 178.24,169.26,140.73,140.54,124.92,122.52,120.01,105.10,79.04,74.24,38.30$, 24.06, 17.04. Analysis: Calcd (\%) for $\mathrm{C}_{13} \mathrm{H}_{13} \mathrm{BrN}_{2} \mathrm{OS}$ : C, 48.01; H, $4.03 \mathrm{~N}, 8.61$; S, 9.86. Found: C, 47.96; H, 3.99; N, 8.39; S, 9.72.

6-Acetylamino-3-allyl-2-methylbenzothiazol-3-ium bromide (6b). Yield 64\%. M.p. 194-196 ${ }^{\circ} \mathrm{C} .{ }^{1} \mathrm{H}$ NMR (300 MHz, DMSO) $\delta 10.66$ (s, 1H, NH), 8.89 (d, J=1.5 Hz, 1H, H-7), 8.22 (d, $J=9.3 \mathrm{~Hz}, 1 \mathrm{H}, \mathrm{H}-4), 7.88$ (dd, $J=9.3,1.5 \mathrm{~Hz}, 1 \mathrm{H}, \mathrm{H}-5), 6.07$ (ddt, $J=17.3,10.5,5.3 \mathrm{~Hz}, 1 \mathrm{H}$, $\left.\mathrm{C} \underline{H}=\mathrm{CH}_{2}\right), 5.40\left(\mathrm{~d}, J=5.3 \mathrm{~Hz}, 2 \mathrm{H}, \mathrm{N}-\mathrm{CH}_{2}\right), 5.37\left(\mathrm{~d}, J_{\text {cis }}=10.5 \mathrm{~Hz}, 1 \mathrm{H},=\mathrm{C} \underline{\mathrm{HH}}\right), 5.34\left(\mathrm{~d}, J_{\text {trans }}=\right.$ $17.3 \mathrm{~Hz}, 1 \mathrm{H},=\mathrm{CH} \underline{\mathrm{H}}), 3.17\left(\mathrm{~s}, 3 \mathrm{H}, \mathrm{CH}_{3}\right), 2.15\left(\mathrm{~s}, 3 \mathrm{H}, \mathrm{COCH}_{3}\right) .{ }^{13} \mathrm{C} \mathrm{NMR}(75 \mathrm{MHz}, \mathrm{DMSO}) \delta$ 175.85, 168.97, 139.00, 136.12, 129.79, 129.39, 120.89, 119.88, 116.89, 112.78, 50.96, 23.98, 16.63. Analysis: Calcd (\%) for $\mathrm{C}_{13} \mathrm{H}_{15} \mathrm{BrN}_{2} \mathrm{OS}$ : C, 47.71; H, $4.62 \mathrm{~N}, 8.56$; S, 9.83. Found: C, $47.81 ; \mathrm{H}, 4.55 ; \mathrm{N}, 8.44 ; \mathrm{S}, 9.68$.

6-Acetylamino-2-methyl-3-propargyl-benzothiazol-3-ium bromide (6c). Yield 60\%,.M.p. 195-196.5 ${ }^{\circ} \mathrm{C} .{ }^{1} \mathrm{H}$ NMR (300 MHz, DMSO) $\delta 10.60$ (s, 1H, NH), 8.90 (d, J=1.8 Hz, 1H, H-7), 8.28 (d, $J=9.3 \mathrm{~Hz}, 1 \mathrm{H}, \mathrm{H}-4), 7.88$ (dd, $J=9.3,1.8 \mathrm{~Hz}, 1 \mathrm{H}, \mathrm{H}-5), 5.70$ (d, $J=2.4 \mathrm{~Hz}, 2 \mathrm{H}, \mathrm{CH}_{2}$ ), $3.83(\mathrm{t}, J=2.4 \mathrm{~Hz}, 1 \mathrm{H}, \equiv \mathrm{CH}), 3.21\left(\mathrm{~s}, 3 \mathrm{H}, \mathrm{CH}_{3}\right), 2.15\left(\mathrm{~s}, 3 \mathrm{H}, \mathrm{COCH}_{3}\right) .{ }^{13} \mathrm{C} \mathrm{NMR}(75 \mathrm{MHz}$, DMSO) $\delta 176.15,168.76,138.92,135.40,129.69,120.83,116.56,112.69,78.66,74.32,39.45$, 23.76, 16.51. Analysis: Calcd (\%) for $\mathrm{C}_{13} \mathrm{H}_{13} \mathrm{BrN}_{2} \mathrm{OS}$ : C, 48.01; H, $4.03 \mathrm{~N}, 8.61$; S, 9.86. Found: C, 47.86; H, 4.00; N, 8.56; S, 9.69. 
Condensation reaction of 4-substituted benzaldehydes with 3-alkyl-2-methyl-5-(or 6-) substituted benzothiazol-3-ium halides. General procedure

A mixture of 5- or 6-substituted-3-alkyl-2-methylbenzothiazol-3-ium halide (5 mmol) the corresponding aldehyde $(5 \mathrm{mmol})$ and pyridine $(0.3 \mathrm{mmol})$ in $5 \mathrm{ml}$ methanol was refluxed for 8-12 h. After slow cooling to r.t. the formed crystalline product was filtered, washed with dry acetone and dried to afford compounds $7 \mathbf{b}-\mathbf{7 e}, \mathbf{8 b}-\mathbf{8 f}$ (Scheme 2).

2-\{(E)-2-[4-(Dimethylamino)phenyl]vinyl\}-3-methyl-5-nitrobenzothiazol-3-ium iodide (7a) was prepared according to procedure described in literature. M. p. $\left(242-243{ }^{\circ} \mathrm{C}\right)$ and NMR data matched the previously published values. ${ }^{13}$ UV/VIS (methanol) $\lambda_{\max }(\varepsilon): 548$ (58 800).

2-\{(E)-2-[4-(Methoxy)phenyl]vinyl\}-3-methyl-5-nitrobenzothiazol-3-ium iodide (7b). Yield $81 \%$. M.p. $217-220{ }^{\circ} \mathrm{C} .{ }^{1} \mathrm{H}$ NMR (300 MHz, DMSO) $\delta 9.06$ (d, $\left.J=2.1 \mathrm{~Hz}, 1 \mathrm{H}, \mathrm{H}-4\right), 8.67$ (d, $J=9.0 \mathrm{~Hz}, 1 \mathrm{H}, \mathrm{H}-7), 8.58$ (dd, $J=9.0,2.1 \mathrm{~Hz}, 1 \mathrm{H}, \mathrm{H}-6), 8.34$ (d, $J=15,9 \mathrm{~Hz}, 1 \mathrm{H}, \mathrm{H}-\mathrm{b}), 8.11$ (d, $\left.J=8.7 \mathrm{~Hz}, 2 \mathrm{H}, \mathrm{H}-2^{\prime}, 6^{\prime}\right), 7.94$ (d, $\left.J=15,9 \mathrm{~Hz}, 1 \mathrm{H}, \mathrm{H}-\mathrm{a}\right), 7.17$ (d, $\left.J=8.7 \mathrm{~Hz}, 2 \mathrm{H}, \mathrm{H}-3^{\prime}, 5^{\prime}\right), 4.41$ $\left(\mathrm{s}, 3 \mathrm{H}, \mathrm{CH}_{3}\right) 3.90\left(\mathrm{~s}, 3 \mathrm{H}, \mathrm{OCH}_{3}\right) .{ }^{13} \mathrm{C} \mathrm{NMR}(75 \mathrm{MHz}, \mathrm{DMSO}) \delta 175.32,163.36,150.78,147.71$, 142.28, 133.92, 132.62, 126.63, 125.57, 122.19, 114.90, 112.19, 110.99, 55.71, 36.67. Analysis: Calcd (\%) for $\mathrm{C}_{17} \mathrm{H}_{15} \mathrm{IN}_{2} \mathrm{O}_{3} \mathrm{~S}: \mathrm{C}, 44.95 ; \mathrm{H}, 3.33 ; \mathrm{N}, 6.17 ; \mathrm{S}, 7.06$. Found: $\mathrm{C}, 44.52 ; \mathrm{H}, 3.17 ; \mathrm{N}$, 6.19; S, 7.17. UV/VIS (methanol) $\lambda_{\max }(\varepsilon): 424$ (13 600).

\section{5-Acetamino-3-allyl-2-\{(E)-2-[4-(dimethylamino)phenyl]vinyl $\}$-benzothiazol-3-ium}

bromide (7c). Yield 75 \%. M.p. 257-258 ${ }^{\circ} \mathrm{C} .{ }^{1} \mathrm{H}$ NMR (300 MHz, DMSO) $\delta 10.56$ (s, 1H, NH), 8.41 (d, 1H, $J=1.5 \mathrm{~Hz}, \mathrm{H}-4), 8.20$ (d, $J=9.0 \mathrm{~Hz}, 1 \mathrm{H}, \mathrm{H}-7), 8.05$ (d, $J=15.3 \mathrm{~Hz}, 1 \mathrm{H}, \mathrm{H}-\mathrm{b}), 7.89$ $\left(\mathrm{d}, J=9.0 \mathrm{~Hz}, 2 \mathrm{H}, \mathrm{H}-2^{\prime}, 6^{\prime}\right), 7.68(\mathrm{dd}, J=9.0,1.5 \mathrm{~Hz}, 1 \mathrm{H}, \mathrm{H}-6), 7.58$ (d, $\left.J=15.3 \mathrm{~Hz}, 1 \mathrm{H}, \mathrm{H}-\mathrm{a}\right)$, $6.81\left(\mathrm{~d}, J=9.0 \mathrm{~Hz}, 2 \mathrm{H}, \mathrm{H}-3^{\prime}, 5^{\prime}\right), 6.07$ (ddt, $\left.J=17.1,10.8,4.2 \mathrm{~Hz}, 1 \mathrm{H}, \mathrm{C} \underline{H}=\mathrm{CH}_{2}\right), 5.37$ (s, 2H, $\left.\mathrm{N}-\mathrm{CH}_{2}\right), 5.36\left(\mathrm{~d}, J_{\text {cis }}=10.8 \mathrm{~Hz}, 1 \mathrm{H},=\mathrm{CHH}\right), 5.22\left(\mathrm{~d}, J_{\text {trans }}=17.1 \mathrm{~Hz}, 1 \mathrm{H},=\mathrm{CH} \underline{\mathrm{H}}\right), 3.10(\mathrm{~s}, 6 \mathrm{H}$, $\left.\mathrm{N}\left(\mathrm{CH}_{3}\right)_{2}\right), 2.13\left(\mathrm{~s}, 3 \mathrm{H}, \mathrm{COCH}_{3}\right) .{ }^{13} \mathrm{C} \mathrm{NMR}(75 \mathrm{MHz}, \mathrm{DMSO}) \delta 171.86,169.05,153.44,150.25$, $141.39,140.15,132.88,130.16,123.99,121.35,120,53,118,88,111,84,105,63,104.62,49,88$, 39.39, 24.05. Analysis: Calcd (\%) for $\mathrm{C}_{22} \mathrm{H}_{24} \mathrm{BrN}_{3} \mathrm{OS}$ : C, 57.64; H, 5.28; N, 9.17; S, 6.99. Found: C, 57.51; H, 5.27; N, 9.19; S, 7.07. UV/VIS (methanol) $\lambda_{\max }(\varepsilon): 539$ (95 400).

5-Acetamino-2-\{(E)-2-[4-(dimethylamino)phenyl]vinyl\}-3-propargylbenzothiazol-3-ium bromide (7d). Yield 80 \%. M.p. 260-262 ${ }^{\circ} \mathrm{C} .{ }^{1} \mathrm{H}$ NMR (300 MHz, DMSO) $\delta 10.58$ (s, 1H, NH), 8.53 (s, 1H, H-4), 8.20 (d, $J=8.7 \mathrm{~Hz}, 1 \mathrm{H}, \mathrm{H}-7), 8.11$ (d, $J=15.0 \mathrm{~Hz}, 1 \mathrm{H}, \mathrm{H}-\mathrm{b}), 7.93$ (d, $J=8.7$ Hz, 2H, H-2', 6'), 7.71 (d, $J=15.0 \mathrm{~Hz}, 1 \mathrm{H}, \mathrm{H}-\mathrm{a}), 7.68$ (dd, $J=8.7,1.5 \mathrm{~Hz}, 1 \mathrm{H}, \mathrm{H}-6), 6.86$ (d, $\left.J=8.7 \mathrm{~Hz}, 2 \mathrm{H}, \mathrm{H}-3^{\prime}, 5^{\prime}\right), 5.67\left(\mathrm{~s}, 2 \mathrm{H}, \mathrm{CH}_{2}\right), 3.76(\mathrm{~s}, 1 \mathrm{H}, \equiv \mathrm{CH}), 3.13\left(\mathrm{~s}, 6 \mathrm{H}, \mathrm{N}\left(\mathrm{CH}_{3}\right)_{2}\right), 2.15(\mathrm{~s}$, $\left.3 \mathrm{H}, \mathrm{COCH}_{3}\right) .{ }^{13} \mathrm{C} \mathrm{NMR}(75 \mathrm{MHz}, \mathrm{DMSO}) \delta 171.72,169.08,153.71,150.99,140.83,140.23$, 133.19 , 124.11, 121.42, 120.21, 119.01, 111.98, 105.18, 104.47, 78.22, 75.59, 38.70, 37.33, 24.06. Analysis: Calcd (\%) for $\mathrm{C}_{22} \mathrm{H}_{22} \mathrm{BrN}_{3} \mathrm{OS}$ : C, 57.90; H, 4.86; N, 9.21; S, 7.03. Found: C, 57.80; H, 4.90; N, 9.19; S, 7.00.UV/VIS (methanol) $\lambda_{\max }(\varepsilon): 547$ (67 500).

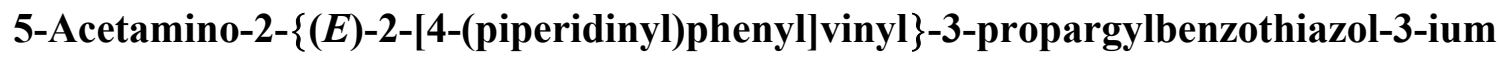
bromide (7e). Yield 73 \%. M.p.224-225 ${ }^{\circ} \mathrm{C} .{ }^{1} \mathrm{H}$ NMR (300 MHz, DMSO) $\delta 10.57$ (s, 1H, NH), 8.54 (d, $J=1.2 \mathrm{~Hz}, 1 \mathrm{H}, \mathrm{H}-4), 8.21$ (d, $J=9.0 \mathrm{~Hz}, 1 \mathrm{H}, \mathrm{H}-7), 8.10$ (d, $J=15.0 \mathrm{~Hz}, 1 \mathrm{H}, \mathrm{H}-\mathrm{b}), 7.92$ $\left(\mathrm{d}, J=9.0 \mathrm{~Hz}, 2 \mathrm{H}, \mathrm{H}-2^{\prime}, 6^{\prime}\right), 7.74$ (d, $\left.J=15.0 \mathrm{~Hz}, 1 \mathrm{H}, \mathrm{H}-\mathrm{a}\right), 7.68$ (dd, $\left.J=9.0,1.5 \mathrm{~Hz}, 1 \mathrm{H}, \mathrm{H}-5\right)$, 
7.08 (d, $\left.J=9.0 \mathrm{~Hz}, 2 \mathrm{H}, \mathrm{H}-3^{\prime}, 5^{\prime}\right), 5.68\left(\mathrm{~s}, 2 \mathrm{H}, \mathrm{CH}_{2}\right), 3.75$ (s, 1H, $\left.\equiv \mathrm{CH}\right), 3.54$ (bs, 4H, $\left.\mathrm{CH}_{2}\right), 2.15$ $\left(\mathrm{s}, 3 \mathrm{H}, \mathrm{COCH}_{3}\right), 1.63-1.60\left(\mathrm{~m}, 6 \mathrm{H}, \mathrm{CH}_{2}\right) .{ }^{13} \mathrm{C} \mathrm{NMR}(75 \mathrm{MHz}, \mathrm{DMSO}) \delta 171.84,169.09$, $153.68,150.59,140.87,140.27,137.38 ; 133.33,124.18,122.10,120.30,119.12,113.32,105.77$, 78.26, 75.56, 47.44, 37.47; 25.06, 24.07, 23.84. Analysis: Calcd (\%) for $\mathrm{C}_{25} \mathrm{H}_{26} \mathrm{BrN}_{3} \mathrm{OS}$ : C, 60.48; H, 5.28; N, 8.46; S, 6.46. Found: C, 60.00; H, 5.16; N, 8.37; S, 6.38. UV/VIS (methanol) $\lambda_{\max }(\varepsilon): 553$ (31 100).

2-\{(E)-2-[4-(Dimethylamino)phenyl]vinyl\}-3-methyl-6-nitrobenzothiazol-3-ium iodide (8a) was prepared according to procedure described in literature. M. p. $\left(257-258^{\circ} \mathrm{C}\right)$ and NMR data matched the previously published values. ${ }^{13}$ UV/VIS (methanol) $\lambda_{\max }(\varepsilon): 562$ (32 700).

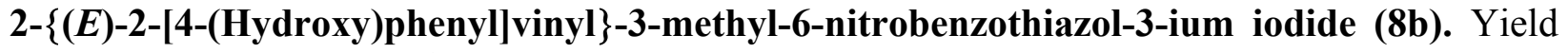
89\%. M.p. 286-287,5 ${ }^{\circ} \mathrm{C}$. ${ }^{1} \mathrm{H}$ NMR (300 MHz, DMSO) $\delta 10.81$ (s, 1H, OH), 9.39 (d, J=2.2 Hz, $1 \mathrm{H}, \mathrm{H}-7), 8.63$ (dd, $J=9.3,2.2 \mathrm{~Hz}, 1 \mathrm{H}, \mathrm{H}-5), 8.38$ (d, $J=9.3 \mathrm{~Hz}, 1 \mathrm{H}, \mathrm{H}-4), 8.34$ (d, $J=16.2 \mathrm{~Hz}$, 1H, H-b), 8.03 (d, $\left.J=8.8 \mathrm{~Hz}, 2 \mathrm{H}, \mathrm{H}-2^{\prime}, 6^{\prime}\right), 7.85$ (d, $\left.J=16.2 \mathrm{~Hz}, 1 \mathrm{H}, \mathrm{H}-\mathrm{a}\right), 6.95$ (d, $J=8.8 \mathrm{~Hz}$, $\left.2 \mathrm{H}, \mathrm{H}-3^{\prime}, 5^{\prime}\right), 4.33$ (s, 3H, $\left.\mathrm{CH}_{3}\right) .{ }^{13} \mathrm{C} \mathrm{NMR}(75 \mathrm{MHz}, \mathrm{DMSO}) \delta 176.36,162.93,151.92,145.87$, 145.76, 133.34, 128.33, 125.43, 124.21, 120.68, 117.33, 116.46, 110.05, 36.60. Analysis: Calcd (\%) for $\mathrm{C}_{16} \mathrm{H}_{13} \mathrm{IN}_{2} \mathrm{O}_{3} \mathrm{~S}$ : C, 43.65; H, 2.98; N, 6.36; S, 7.28. Found: C, 43.56; H, 3.12; N, 6.52; S, 7.45. UV/VIS (methanol) $\lambda_{\max }(\varepsilon): 568$ (10 700).

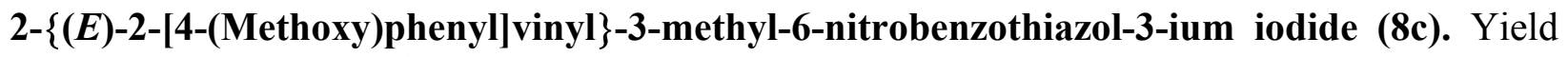
95\%. M.p. 232-233 ${ }^{\circ} \mathrm{C} .{ }^{1} \mathrm{H}$ NMR (300 MHz, DMSO) $\delta 9.43$ (d, $J=2.4 \mathrm{~Hz}, 1 \mathrm{H}, \mathrm{H}-7$ ), 8.66 (dd, $J=9.0,2.4 \mathrm{~Hz}, 1 \mathrm{H}, \mathrm{H}-5), 8.42$ (d, $J=9.0 \mathrm{~Hz}, 1 \mathrm{H}, \mathrm{H}-4), 8.39$ (d, $J=15.9 \mathrm{~Hz}, 1 \mathrm{H}, \mathrm{H}-\mathrm{b}), 8.15$ (d, $\left.J=9.0 \mathrm{~Hz}, 2 \mathrm{H}, \mathrm{H}-2^{\prime}, 6^{\prime}\right), 7.94$ (d, $\left.J=15.9 \mathrm{~Hz}, 1 \mathrm{H}, \mathrm{H}-\mathrm{a}\right), 7.20$ (d, $\left.J=9.0 \mathrm{~Hz}, 2 \mathrm{H}, \mathrm{H}-3^{\prime}, 5^{\prime}\right), 4.37$ $\left(\mathrm{s}, 3 \mathrm{H}, \mathrm{CH}_{3}\right), 3.91$ (s, 3H, $\left.\mathrm{OCH}_{3}\right) .{ }^{13} \mathrm{C} \mathrm{NMR}(75 \mathrm{MHz}, \mathrm{DMSO}) \delta 176.50,163.56,151.30,145.98$, $145.74,132.85,128.49,126.82,124.28,120.78,117.53,115.03,111.25,55.85,37.23$. . Analysis: Calcd (\%) for $\mathrm{C}_{17} \mathrm{H}_{15} \mathrm{IN}_{2} \mathrm{O}_{3} \mathrm{~S}$ : C, 44.95; H, 3.33; N, 6.17; S, 7.06. Found: C, 45.00; H, 3.35; N, 6.14; S, 7.00. UV/VIS (methanol) $\lambda_{\max }(\varepsilon)$ : 432 (18 800).

\section{6-Acetamino-3-allyl-2-\{(E)-2-[4-(dimethylamino)phenyl]vinyl $\}$-benzothiazol-3-ium}

bromide (8d). Yield 87\%. M.p. 276-277 ${ }^{\circ} \mathrm{C} .{ }^{1} \mathrm{H}$ NMR (300 MHz, DMSO) $\delta 10.48$ (s, 1H, NH), 8.71 (d, $J=1.9 \mathrm{~Hz}, 1 \mathrm{H}, \mathrm{H}-7), 8.02$ (d, $J=15.2 \mathrm{~Hz}, 1 \mathrm{H}, \mathrm{H}-\mathrm{b}), 8.00$ (d, $J=9.1 \mathrm{~Hz}, 1 \mathrm{H}, \mathrm{H}-4), 7.88$ $\left(\mathrm{d}, J=9.1 \mathrm{~Hz}, 2 \mathrm{H}, \mathrm{H}-2^{\prime}, 6^{\prime}\right), 7.71(\mathrm{dd}, J=9.1,1.9 \mathrm{~Hz}, 1 \mathrm{H}, \mathrm{H}-5), 7.55$ (d, $\left.J=15.2 \mathrm{~Hz}, 1 \mathrm{H}, \mathrm{H}-\mathrm{a}\right)$, 6.84 (d, $\left.J=9.1 \mathrm{~Hz}, 2 \mathrm{H}, \mathrm{H}-3^{\prime}, 5^{\prime}\right), 6.05$ (ddt, $J=17.6,10.4,4.5 \mathrm{~Hz}, 1 \mathrm{H}, \mathrm{C} \underline{H}=\mathrm{CH}_{2}$ ), 5.45 (d, $\left.J=4.5 \mathrm{~Hz}, 2 \mathrm{H}, \mathrm{N}-\mathrm{CH}_{2}\right), 5.32\left(\mathrm{~d}, J_{\text {cis }}=10.4 \mathrm{~Hz}, 1 \mathrm{H},=\mathrm{CHH}\right), 5.25\left(\mathrm{~d}, J_{\text {trans }}=17.6 \mathrm{~Hz}, 1 \mathrm{H}\right.$, $=\mathrm{CH} \underline{\mathrm{H}}), 3.11\left(\mathrm{~s}, 6 \mathrm{H}, \mathrm{N}\left(\mathrm{CH}_{3}\right)_{2}\right), 2.13\left(\mathrm{~s}, 3 \mathrm{H}, \mathrm{COCH}_{3}\right) \cdot{ }^{13} \mathrm{C} \mathrm{NMR}(75 \mathrm{MHz}, \mathrm{DMSO}) \delta 169.85$, $168.80,153.36,149.75,138.58,136.42,132.64,130.56,127.95,121.37,120.35,118.79,116.07$, 112.56, 111.86, 105.80, 49.73, 40.30, 23.97. Analysis: Calcd (\%) for $\mathrm{C}_{22} \mathrm{H}_{24} \mathrm{BrN}_{3} \mathrm{OS}$ : C, 57.64; H, 5.28; N, 9.17; S, 6.99. Found: C, 57.95; H, 5.27; N, 6.52; S, 6.91. UV/VIS (methanol) $\lambda_{\max }$ (ع): 535 (80 700).

6-Acetamino-2-\{(E)-2-[4-(dimethylamino)phenyl]vinyl $\}$-3-propargylbenzothiazol-3-ium bromide (8e). Yield 79 \%. M.p. 267-268 ${ }^{\circ} \mathrm{C} .{ }^{1} \mathrm{H}$ NMR (300 MHz, DMSO) $\delta 10.53$ (s, 1H, NH), 8.72 (d, $J=1.5 \mathrm{~Hz}, 1 \mathrm{H}, \mathrm{H}-7), 8.07$ (d, $J=14.7 \mathrm{~Hz}, 1 \mathrm{H}, \mathrm{H}-\mathrm{b}), 8.06$ (d, $J=9.1 \mathrm{~Hz}, 1 \mathrm{H}, \mathrm{H}-4), 7.92$ $\left(\mathrm{d}, J=9.0 \mathrm{~Hz}, 2 \mathrm{H}, \mathrm{H}-2^{\prime}, 6^{\prime}\right), 7.77$ (dd, $\left.J=9.1,1.5 \mathrm{~Hz}, 1 \mathrm{H}, \mathrm{H}-5\right), 7.68$ (d, $\left.J=15.0 \mathrm{~Hz}, 1 \mathrm{H}, \mathrm{H}-\mathrm{a}\right)$, 
$6.87\left(\mathrm{~d}, J=9.0 \mathrm{~Hz}, 2 \mathrm{H}, \mathrm{H}-3^{\prime}, 5^{\prime}\right), 5.70\left(\mathrm{~s}, 2 \mathrm{H}, \mathrm{CH}_{2}\right), 3.74(\mathrm{~s}, 1 \mathrm{H}, \equiv \mathrm{CH}), 3.14\left(\mathrm{~s}, 6 \mathrm{H}, \mathrm{N}\left(\mathrm{CH}_{3}\right)_{2}\right)$, $2.15\left(\mathrm{~s}, 3 \mathrm{H}, \mathrm{COCH}_{3}\right) .{ }^{13} \mathrm{C} \mathrm{NMR}(75 \mathrm{MHz}, \mathrm{DMSO}) \delta 169.98,168.96,153.73,150.63,150.62$, 138.78, 135.91, 133.11, 133.07, 127.78, 121.53, 115.91, 112.09, 111.06 78.19, 76.03, 43.74, 24.09, 22.19. Analysis: Calcd (\%) for $\mathrm{C}_{22} \mathrm{H}_{22} \mathrm{BrN}_{3} \mathrm{OS}$ : C, 57.90; H, 4.86; N, 9.21; S, 7.03. Found: C, 57.76; H, 4.81; N, 9.17; S, 7.06. UV/VIS (methanol) $\lambda_{\max }(\varepsilon): 544$ (44 200).

\section{6-Acetamino-2-\{(E)-2-[4-(piperidinyl)phenyl]vinyl\}-3-propargylbenzothiazol-3-ium}

bromide (8f). Yield 77 \%. M.p. 276-278 ${ }^{\circ} \mathrm{C} .{ }^{1} \mathrm{H}$ NMR (300 MHz, DMSO) $\delta 10.52$ (s, 1H, NH), 8.72 (d, $J=1.8 \mathrm{~Hz}, 1 \mathrm{H}, \mathrm{H}-7), 8.07$ (d, $J=9.0 \mathrm{~Hz}, 1 \mathrm{H}, \mathrm{H}-4), 8.05$ (d, $J=15.0 \mathrm{~Hz}, 1 \mathrm{H}, \mathrm{H}-\mathrm{b}), 7.90$ (d, $\left.J=9.0 \mathrm{~Hz}, 2 \mathrm{H}, \mathrm{H}-2^{\prime}, 6^{\prime}\right), 7.77$ (dd, $\left.J=9.0,1.8 \mathrm{~Hz}, 1 \mathrm{H}, \mathrm{H}-5\right), 7.71$ (d, $\left.J=15.0 \mathrm{~Hz}, 1 \mathrm{H}, \mathrm{H}-\mathrm{a}\right)$, 7.07 (d, $\left.J=9.0 \mathrm{~Hz}, 2 \mathrm{H}, \mathrm{H}-3^{\prime}, 5^{\prime}\right), 5.74\left(\mathrm{~d}, J=1.8 \mathrm{~Hz}, 2 \mathrm{H}, \mathrm{CH}_{2}\right), 3.74(\mathrm{~d}, J=1.8 \mathrm{~Hz}, 1 \mathrm{H}, \equiv \mathrm{CH})$, $3.54\left(\mathrm{~m}, 4 \mathrm{H}, \mathrm{CH}_{2}\right), 2.13\left(\mathrm{~s}, 3 \mathrm{H}, \mathrm{COCH}_{3}\right), 1.63\left(\mathrm{~m}, 6 \mathrm{H}, \mathrm{CH}_{2}\right) .{ }^{13} \mathrm{C} \mathrm{NMR}(75 \mathrm{MHz}, \mathrm{DMSO}) \delta$ $170.05,168.96,153.71,150.18,138.86,135.92,133.120,133.16,127.88,122.25,115.99$, 113.48, $112.80106 .10,78.23,76.00,47.57,38.36,25.14,24.10,23.95$. Analysis: Calcd (\%) for $\mathrm{C}_{25} \mathrm{H}_{26} \mathrm{BrN}_{3} \mathrm{OS}$ : C, 60.48; H, 5.28; N, 8.46; S, 6.46. Found: C, 60.13; H, 5.28; N, 8.52; S, 6.45. UV/VIS (methanol) $\lambda_{\max }(\varepsilon): 549$ (65 600).

\section{Antimicrobial activities}

The final compounds 7a-7e, 8a-8f have been tested in vitro for their antibacterial activity against four strains of Gram-positive bacteria: Staphylococcus aureus, Bacillus subtilis, Micrococcus luteus and Enterococcus faecalis. The standard method (Mueller-Hinton agar, cultivation at 37 ${ }^{\circ} \mathrm{C}$ for 18 h. ${ }^{17}$ was used. The tested compounds were dissolved in DMSO, then diluted with water. The screening against four strains of yeast microorganisms: Saccharomyces cerevisiae, Hansenula anomala, Candida albicans CCY 29-3-112, C. albicans 271 was also carried out. The classical screening ${ }^{18}$ (static cultivation at $28{ }^{\circ} \mathrm{C}$ for $24 \mathrm{~h}$., Saboraud medium) has been realised. DMSO as a co-solvent was used.

The results of biological screening are presented in Tables 1 and 2. Values of minimum inhibitory concentrations ( $\mathrm{MIC}$ in $\mu \mathrm{g} / \mathrm{ml}$ ) are given.

Table 1. Antimicrobial activities against yeasts and Gram-positive bacteria (MIC in $\mu \mathrm{g} / \mathrm{ml}$ )

\begin{tabular}{cccccccccccc}
\hline & $\mathbf{7 a}$ & $\mathbf{7 b}$ & $\mathbf{7 c}$ & $\mathbf{7 d}$ & $\mathbf{7 e}$ & $\mathbf{8 a}$ & $\mathbf{8 b}$ & $\mathbf{8 c}$ & $\mathbf{8 d}$ & $\mathbf{8 e}$ & $\mathbf{8 f}$ \\
\hline $\begin{array}{c}\text { Candida albicans } 271 \\
\text { C. albicans CCY 29-3-112 }\end{array}$ & - & - & - & - & - & - & - & - & 250 & - & - \\
$\quad \begin{array}{l}\text { S. cerevisiae } \\
\text { H. anomala }\end{array}$ & 125 & 250 & - & - & - & 125 & - & 250 & - & - & - \\
$\quad$ S. aureus & - & - & - & - & - & & - & - & - & - & - \\
M. luteus & - & - & - & - & - & - & - & - & 125 & - & - \\
E. faecalis & - & 125 & 125 & 250 & 31 & - & - & 250 & - & 250 & - \\
B. subtilis & - & - & - & - & - & - & - & - & - & - & - \\
\end{tabular}


Table 2. The comparison of the influence of substituents in position 5- and 6- to the biological activities

\begin{tabular}{lllcccc}
\hline & \multicolumn{1}{c}{$5-\mathrm{Z} / 6-\mathrm{Z}$} & \multicolumn{1}{c}{$\mathrm{Y}$} & C.albicans & S. cerevisiae & B. subtilis & M. luteus \\
\hline $\mathbf{7 a} / \mathbf{8 a}$ & $-\mathrm{NO}_{2}$ & $-\mathrm{N}\left(\mathrm{CH}_{3}\right)_{2}$ & $-/-$ & $125 / 125$ & $-/-$ & $-/-$ \\
$\mathbf{7 b} / \mathbf{8 c}$ & $-\mathrm{NO}_{2}$ & $-\mathrm{O}-\mathrm{CH}_{3}$ & $-/-$ & $250 /-$ & $-/-$ & $125 / 250$ \\
$\mathbf{7 c / 8 d}$ & $-\mathrm{NH}-\mathrm{CO}-\mathrm{CH}_{3}$ & $-\mathrm{N}\left(\mathrm{CH}_{3}\right)_{2}$ & $-/ 250$ & $-/-$ & $31 / 250$ & $125 /-$ \\
$\mathbf{7 d} / \mathbf{8 e}$ & $-\mathrm{NH}-\mathrm{CO}-\mathrm{CH}_{3}$ & $-\mathrm{N}\left(\mathrm{CH}_{3}\right)_{2}$ & $-/-$ & $-/-$ & $250 /-$ & $250 /-$ \\
$7 \mathbf{7 e} / \mathbf{8 f}$ & $-\mathrm{NH}-\mathrm{CO}-\mathrm{CH}_{3}$ & $-\mathrm{N}$ & $-/-$ & $-/-$ & $31 / 125$ & $31 / 250$ \\
\hline
\end{tabular}

\section{Conclusions}

Eleven benzothiazolium derivatives substituted in position 5- and/or 6- on heterocyclic ring have been prepared by the condensation reaction. The compounds have been tested against Grampositive bacteria and yeast microorganisms.

The prepared compounds show only moderate biological activity. The activity of the compounds with electronacceptor $\mathrm{NO}_{2}$ substituent is not significant neither against Grampositive bacteria nor yeast microorganisms.

The compounds bearing electrondonor substituent $-\mathrm{NH}-\mathrm{CO}-\mathrm{CH}_{3}$ are not active against yeasts but compounds 7e and 7e show enhanced activity against Bacillus subtilis and 7e is active also against Micrococcus luteus. To evaluate the efficiency of the different positions on benzothiazole ring we compare appropriate pairs of isomeric benzothiazoles. The substituent at the position 5has more significant effect.

The compound 7e seems to be the most active one against Gram-positive bacteria but the main contribution to the activity comes probably from piperidine moiety on benzene ring.

The analysis of UV-vis spectra of prepared compounds under this study confirms their pushpull character expressed by the long-wave charge-transfer band in the visible region. The compounds with amino groups as electron-donor substituent and $\mathrm{NO}_{2}$ group in position 6- of the benzothiazolium ring are potential candidates for nonlinear optical application.

\section{Acknowledgements}

The financial support for this research was granted by the Slovak Grant Agency (Grant VEGA $1 / 4470 / 07)$ and is gratefully acknowledged. 


\section{References}

1. Kashiyama, E.; Hutchinson, I.; Chua, M. S.; Stinson, S. F.; Philips, L. R.; Kaur, G.; Sansville, E. A.; Bradshaw, T. D.; Westwell, A. D.; Stevens M. F. G. J. Med. Chem. 1999, 42, 4172.

2. Batista, R. M. F.; Costa, S. P. G.; Malheiro, E. L.; Belsley, M.; Raposo, M. M. M. Tetrahedron 2007, 63, 4258.

3. Reddy, P. V. G.; Lin, Y.-W.; Chang, H.-T. ARKIVOC 2007, (xvi), 113.

4. Zahradník, P.; Foltínová, P.; Halgaš, J. SAR and QSAR in Envir.Res. 1996, 5, 51; Chem. Abstr. 1996, 124, 281374.

5. Hatrik, S.; Zahradník P. J. Chem. Inf. Comput. Sci. 1996, 36, 992; Chem. Abstr. 1996, 125, 160654.

6. Magdolen, P.; Zahradnik, P.; Foltinova, P. Pharmazie 2000, 55, 803.

7. Magdolen, P.; Zahradnik, P.; Foltinova, P. Arzneim.-Forsch./Drug.Res. 2000, 5, 1023, Chem. Abstr. 2001, 134, 160079.

8. Zahradník, P. Chem. Papers 1990, 44, 145; Chem. Abstr. 1990, 113, 114402.

9. Mizuno, S.; Adachi, K. J. J. Pharm. Soc. Japan 1952, 72, 745; Chem. Abstr. 1954, 48, 2689.

10. Spieler, I.; Prijs, B. Helv. Chim. Acta 1950, 33, 1429.

11. Clarke, K.; Hughes, C. G.; Scrowston, R. M.: J. Chem. Soc., Perkin Trans. I 1973, 356.

12. Hrobárik, P.; Sigmundová, I.; Zahradník, P. Synthesis 2005, 600.

13. Sigmundová, I.; Zahradník, P.; Loos, D. Collect. Czech. Chem. Commun. 2007, 72, 1057.

14. Cuadro, A.; Perez-Butragueno, J.; Pastor-Maeso, M.; Alvarez-Builla, J. Il Farmaco 1992, $47,477$.

15. Jones, E. J.; Dauschroder, R. E. U.S. Pat. 2759 821; Chem. Abstr. 1957, 51, 117.

16. Dryanska, V., Ivanov, Ch. Dokl. Bulg. Akad. Nauk 23 1970, 1227; Chem. Abstr. 1971, 74, 31706.

17. National Committee for Clinical Laboratory Standards: Methods for Dilution Antimicrobial Susceptibility Tests for Bacteria that Grow Aerobically. Approved Standards M7-A2. Villanova, National Committee for Clinical Laboratory Standards 1990.

18. Stevens, D. A. Mycopathol. 1984, 87, 135. 